\title{
Influence of Preparation Conditions of MIL-88A on Catalytic Degradation of Orange G and Dibutyl Phthalate
}

\author{
Jiu-Mei WANG ${ }^{1, a}$, Jin-Quan WAN ${ }^{1, b,{ }^{*}, \text { Yong-Wen MA }}{ }^{1, c}$, Yan WANG ${ }^{1, d}$, and \\ Ze-Yu GUAN ${ }^{1, \text { e }}$
}

${ }^{1}$ School of Environment and Energy, South China University of Technology, Guangzhou 510006, China.

\author{
a13424489831@163.com , bppjqwan@scut.edu.cn, 'ppywma@scut.edu.cn, \\ dyanwang@scut.edu.cn, ${ }^{\mathrm{d}}$ guanzeyul@126.com \\ * Corresponding author
}

Keywords: Preparation conditions, MIL-88A, Orange G, Dibutyl phthalate, Degradation

\begin{abstract}
In this paper, the effect of preparation conditions of the catalyst MIL-88A were investigated. The results shows that MIL-88A with the raw materials proportion of $\mathrm{n}$ (Fumaric acid/ $\mathrm{Fecl}_{3} \cdot 6 \mathrm{H}_{2} \mathrm{O}$ ) $=1: 1$, the synthesis temperature of $85^{\circ} \mathrm{C}$ and crystallization time of $2 \mathrm{~h}$, activation temperature of $100^{\circ} \mathrm{C}$ and drying time of $6 \mathrm{~h}$, which would be achieved the optimal catalytic performance on degradation of orange G (OG) through PS activation. Furthermore, it was the first attempt to apply MIL-88A in removing dibutyl phthalate (DBP) and obtained the ideal result. The removal rate of DBP can reach up to $76.4 \%$ catalyze by MIL-88A\&St-3/Ct-2. It was indicated that MIL-88A with suitable preparation conditions would be a high efficiency catalyst in the application of advanced oxidation processes for degrading contaminants.
\end{abstract}

\section{Introduction}

In recent years, Metal-organic frameworks (MOFs) have attracted more and more attention from both academia and industry. MOFs are composed of transition metal ions and organic ligands, which demonstrate special physical and chemical properties such as high surface area, controllable pore volume, multiple functionalities and high chemical resistance, etc. [1 3]. MOFs have been successfully applied in various fields such as gas storage [4 6], catalysis [7 9], chemical analysis [10], biological medicine [6, 11], and photo catalysis [12]. Many of the adsorption materials synthesized by MOFs can efficiently remove poisonous and harmful substances from polluted water including heavy metals [13], halogens [14], toxic dyes [15], and drugs [16].

Besides, advanced oxidation processes (AOPs) have high efficiency and no pollution after treatment, so it is a prospective technique for treating toxic contaminations in water. MOFs also have been confirmed that it can be used as an efficient heterogeneous catalyst to degrade contaminants in AOPs such as Fenton-like reaction and PS/PMS activation. For example, MIL-100(Fe) and $\left[\mathrm{Cu}_{2} \text { (btec) (btx) }{ }_{1.5}\right]_{\mathrm{n}}$, as a Fenton catalyst, were determined to have high catalytic activity towards $\mathrm{H}_{2} \mathrm{O}_{2}$ [17 18]. Cobalt-based MOF, like ZIF-67 and $\mathrm{Co}_{3}$ (BTC) $2.12 \mathrm{H}_{2} \mathrm{O}$, which were all proved to be good heterogeneous peroxymonosulfate (PMS) catalyst [19 20]. And Fe-based MOF such as MIL-53, Fe-MOF-74 and MIL-101 were also used as a Photo catalysis and 
Fenton reaction catalyst to degrade hazardous substances effectively [21 24].

Herein, we reported a kind of Fe-based MOFs, MIL-88A, used as an efficient catalyst to degrade organic pollutants through per sulfate activation. And in this study, the influence of preparation conditions of MIL-88A, such as different raw materials proportion, synthesis/activation temperature and crystallization/drying time on degradation of pollutants, were the mainly research objects. And those MIL-88A produced in different conditions were used for activating PS to compare the catalytic performance. Furthermore, it was the first attempt to use the MIL-88A to activate PS for removing dibutyl phthalate (DBP) and achieves ideal results. DBP is preferentially chosen because it is one of the most widely used phthalic acid esters (PAEs) and the typical, refractory pollutants in Papermaking Wastewater [25]. Moreover, it is suspected to be toxic and carcinogenic, leading to serious health problems such as peripheral polyneuritis, multiple neuritis and myelo meningitis [26]. In addition, DBP is difficult to destruct by conventional methods because of its stable chemical structure and long chains [27]. For this reason, developing a catalyst for effective catalytic degradation of DBP could be urgent and particularly important.

\section{Experimental}

\section{Reagents and Chemicals}

The commercially available dye Orange G, Fumaric acid, Sodium persulfate dibutyl phthalate (DBP) were obtained from Aladdin Chemical Reagent Co. Ltd, (Shanghai China). Iron chloride hexahydrate $\left(\mathrm{Fecl}_{3} \cdot 6 \mathrm{H}_{2} \mathrm{O}\right)$, Absolute ethanol, methyl alcohol were purchased from Sino pharm Chemical Reagent Co. Ltd, (Beijing China). All above-mentioned reagents and chemicals were analytical grade and used without further purification. The D.I. water used in the experiments was purified by Millipore Milli-Q system.

\section{Synthesis of the Catalysts}

Different raw materials proportion: The catalyst MIL-88A was produced according to the method which was previously reported with certain after-treatment modifications [28]. Basically, Fumaric acid $\left(8.4 \mathrm{mmol}\right.$ ) and different proportions of $\mathrm{Fecl}_{3} \cdot 6 \mathrm{H}_{2} \mathrm{O}$ [n (Fumaric acid/ $\mathrm{Fecl}_{3} \cdot 6 \mathrm{H}_{2} \mathrm{O}$ ) $=1: 1 / 1: 2 /$ 1:3] were added to a beaker with $42 \mathrm{~mL}$ D.I. water. The mixed solutions was stirred for an hour at Magnetic Stirrers before it was placed into a $100 \mathrm{~mL}$ Teflon-lined steel autoclave, then heated at $\mathrm{T}=85^{\circ} \mathrm{C}$ for 2 hours. Until drops to the room temperature, the precipitates washed by ethanol and water repetitively and recovered by centrifugation at $10000 \mathrm{rpm}$ for $10 \mathrm{~min}$. Finally dried in a Vacuum Oven at $100^{\circ} \mathrm{C}$ for 10 hours. These samples were named as MIL-88A\&Rp-1/ Rp-2/ Rp-3 respectively. Another group, $\mathrm{Fecl}_{3} \cdot 6 \mathrm{H}_{2} \mathrm{O}(8.4 \mathrm{mmol})$ and different proportions of Fumaric acid [n $\left(\mathrm{Fecl}_{3} \cdot 6 \mathrm{H}_{2} \mathrm{O} /\right.$ Fumaric acid $)=1: 2 /$ 1:3] participated and marked as MIL-88A\&Rp-4/ Rp-5 respectively. And other conditions were consistent with above.

Different synthesis temperature and crystallization time: change the synthesis temperature to $45^{\circ} \mathrm{C} / 65^{\circ} \mathrm{C} / 85^{\circ} \mathrm{C} / 105^{\circ} \mathrm{C} / 125^{\circ} \mathrm{C} / 145^{\circ} \mathrm{C}$ and mark as MIL-88A\&St-1/ St-2/ St-3/ St-4/ St-5/ St-6 respectively, change the crystallization time to $1 \mathrm{~h} / 2 \mathrm{~h} / 4 \mathrm{~h} / 6 \mathrm{~h} / 8 \mathrm{~h} / 12 \mathrm{~h}$ and name as MIL-88A\&Ct-1/ Ct-2/ Ct-3/ Ct-4/ Ct-5/ Ct-6 respectively. Only variables were different in preparation process of all samples, and other conditions were consistent with above.

Different activation temperature and drying time: change the activation temperature to $60^{\circ} \mathrm{C} /$ $100^{\circ} \mathrm{C} / 140^{\circ} \mathrm{C} / 180^{\circ} \mathrm{C}$ and mark as MIL-88A\&At-1/ At-2/ At-3/ At-4 respectively, change the drying time to $6 \mathrm{~h} / 10 \mathrm{~h} / 14 \mathrm{~h} / 18 \mathrm{~h}$ and name as MIL-88A\&Dt-1/ Dt-2/ Dt-3/ Dt-4 respectively. Only variables were different in preparation process of all samples, and other conditions were consistent 
with above.

\section{Catalytic Degradation Experiment of OG and DBP}

The solution of Orange G, DBP and sodium per sulfate were prepared at first, and then added a certain amount of PS solution and a certain concentration of OG/DBP with a certain volume of water to an Erlenmeyer flask. After that, the catalyst MIL-88A was added to the Erlenmeyer flask which was taken to the Shaking table with speed of 180rpm at room temperature. Sampled at regular intervals and quenched with alcohol immediately. The compounds to be analyzed must go through the membrane (Aperture, $0.22 \mu \mathrm{m}$ ) to remove the solids which could affect the results. Residual dye was analyzed by UV-Vis spectrophotometry method (Tianmei, UV2310-II) at a maximum absorption wavelength $\left(\lambda_{\max }=478 \mathrm{~nm}\right)$. The concentration of DBP was determined using a high performance liquid chromatography (Shimadzu LC-20A) equipped with UV-visible detection at a wavelength of $284 \mathrm{~nm}$, using an Agilent Model Eclipse XDB-C18 column. The injection volume was $10 \mu \mathrm{L}$ and the mobile phase was a mixture of $90 \%$ methanol/ $10 \%$ water at a rate of $0.8 \mathrm{~mL} / \mathrm{min}$ at a controlled temperature of $30^{\circ} \mathrm{C}$. Experiments were repeated at least twice in each group.

\section{Results and Discussion}

\section{The Effect of Raw Materials Proportion of MIL-88A on Degradation of Orange G}

Fumaric acid and $\mathrm{Fecl}_{3} \cdot 6 \mathrm{H}_{2} \mathrm{O}$ were the raw materials of MIL-88A, and the ratio between them should be a very important parameter for the performance of catalyst. MIL-88A used in this work was at a dosage of $0.3 \mathrm{~g} / \mathrm{L}$ and the catalytic activity was investigated by degradation of orange $\mathrm{G}$ (OG). Fig.1 exhibited the removal rates of $O G$ as a function of time in oxidation by different raw materials proportion of MIL-88A. The degradation ability of MIL-88A under investigation in removal of OG was found in the following order: MIL-88A\&Rp-1>Rp-2>Rp-3>Rp-4>Rp-5. It was obvious that raw materials proportion will affect the final results in different extent. This phenomenon might be caused by particle size. With the increase of raw materials added in a same container, the quality of synthesized catalyst and particle size should become more and bigger, and this would lead to a decrease in the activity of the catalyst. The excessive presence of fumaric acid also inhibited the reaction [28], so catalyst with high dosage of fumaric acid such as MIL-88A\&Rp-4 and Rp-5 showed a weaker catalytic performance than others. In summary, MIL-88A with n (Fumaric acid/ $\mathrm{Fecl}_{3} \cdot 6 \mathrm{H}_{2} \mathrm{O}$ ) $=1: 1$ was the best choice.

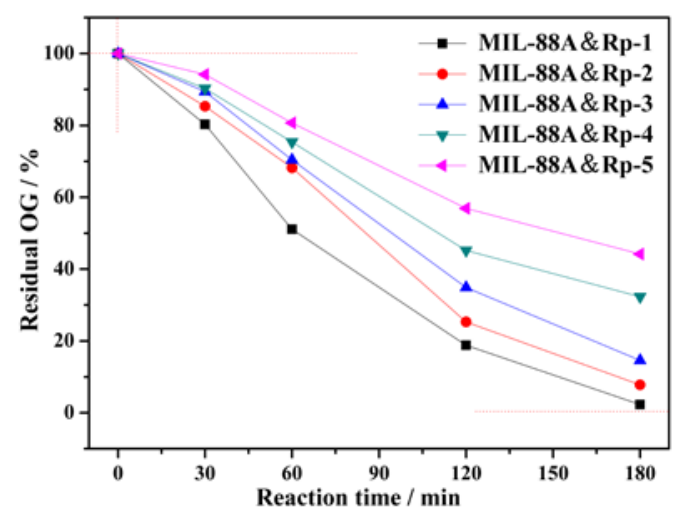

Fig. 1 The effect of raw materials proportion of MIL-88A on degradation of Orange G

(Experimental conditions: $[\mathrm{OG}]=0.2 \mathrm{mmol} / \mathrm{L}$, OG: $\mathrm{PS}=1: 60$, MIL-88A dosage $=0.3 \mathrm{~g} / \mathrm{L}, \mathrm{T}=25^{\circ} \mathrm{C}$, $\mathrm{pH}=3.26$.) 


\section{The Effect of Synthesis Temperature and Crystallization Time of MIL-88A on Degradation of Orange G}

In the preparation of a catalyst, synthesis temperature and crystallization time were very important and could make a big difference of the results. Accordingly, the synthesis temperature of $45^{\circ} \mathrm{C} /$ $65^{\circ} \mathrm{C} / 85^{\circ} \mathrm{C} / 105^{\circ} \mathrm{C} / 125^{\circ} \mathrm{C} / 145^{\circ} \mathrm{C}$ and crystallization time of $1 \mathrm{~h} / 2 \mathrm{~h} / 4 \mathrm{~h} / 6 \mathrm{~h} / 8 \mathrm{~h} / 12 \mathrm{~h}$ were investigated respectively. MIL-88A with synthesis temperature of $85^{\circ} \mathrm{C}$ and crystallization time of $2 \mathrm{~h}$ obtained the optimal performance for catalytic degradation of OG as shown in Fig.2A and Fig.2B. The residual OG first decreases and then increases with the increasing of synthesis temperature of MIL-88A, and this could be caused by the difference of $\mathrm{S}_{\mathrm{BET}}$, dissolved quantity of Fe and pore volume which was proved by Wang et al. [28]. Besides, with the increase of synthesis temperature, the size of samples became larger and the surface of samples were cracked [28]. Therefore, MIL-88A was not suitable produced at high temperature because of the damage the crystal structure. Similarly, numbers of MIL-88A were in growth stage when the crystallization time was short and most of the MIL-88A were already in full-grown stage on the whole field of vision when the crystallization time was longer as shown in SEM reported by Wang et al. [28]. Whether the aging time too long or too short was not conducive to the formation of catalytic materials.
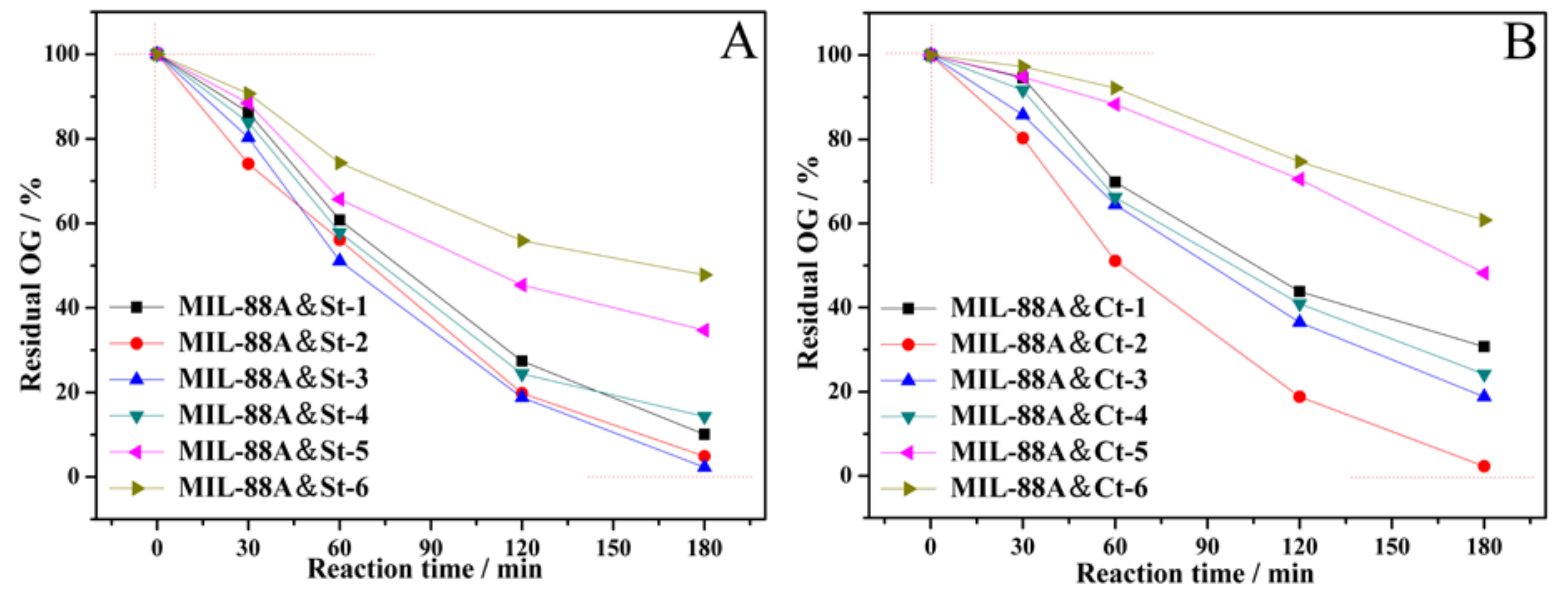

Fig. 2 A. The effect of synthesis temperature of MIL-88A on degradation of Orange G.

B. The effect of crystallization time of MIL-88A on degradation of Orange G.

(Experimental conditions: [OG] $=0.2 \mathrm{mmol} / \mathrm{L}, \mathrm{OG}$ : PS=1:60, MIL-88A dosage $=0.3 \mathrm{~g} / \mathrm{L}, \mathrm{T}=25 \quad{ }^{\circ} \mathrm{C}$, $\mathrm{pH}=3.26$.)

\section{The Effect of Activation Temperature and Drying Time of MIL-88A on Degradation of Orange G}

MIL-88A with activation temperature of $60^{\circ} \mathrm{C} / 100^{\circ} \mathrm{C} / 140^{\circ} \mathrm{C} / 180^{\circ} \mathrm{C}$ and drying time of $6 \mathrm{~h} /$ $10 \mathrm{~h} / 14 \mathrm{~h} / 18 \mathrm{~h}$ were respectively selected to examine the influence of this two parameter on catalytic activity by remove OG. The corresponding decolonization efficiencies were displayed in Fig.3. On the whole, as the activation temperature increased from $60^{\circ} \mathrm{C}$ to $100^{\circ} \mathrm{C}$, the decolorizing effect was obviously improved. But when exceeded $100^{\circ} \mathrm{C}$ such as $140^{\circ} \mathrm{C}$ and $180^{\circ} \mathrm{C}$, the decolorizing rate dropped rapidly. On the other hand, with the increasing of drying time of catalyst, the removal rate of OG showed a decreasing trend. It implies that proper raise activation temperature could promote the catalytic of MIL-88A, but too high activation temperature would 
lead to a change in the structure of catalyst and the catalytic activity decreased. In particular, prolonging the aging time would only have an inhibited effect. This is mainly due to the variation between catalytic activities of MIL-88A with different activation temperature and drying time was in accordance with the changing regulations of relative content of $\mathrm{Fe}^{\mathbb{I}} / \mathrm{Fe}^{\mathrm{II}}$, as proved by $\mathrm{Pu}$ et al. [29] .Theoretically, the increase of relative amount of Fe would afford alternative activate site for PS, thus could enhance the catalytic activity of catalysts [30]. Another possible reason was that the change of $\mathrm{S}_{\mathrm{BET}}$ and total pore volume of catalysts with different vacuum activation conditions [29], which did have certain influences on the catalytic activities of MIL-88A. Therefore, it can be concluded that the catalyst MIL-88A with a suitable activation temperature and drying time could be able to maintain higher activation performance as well as intact structures.

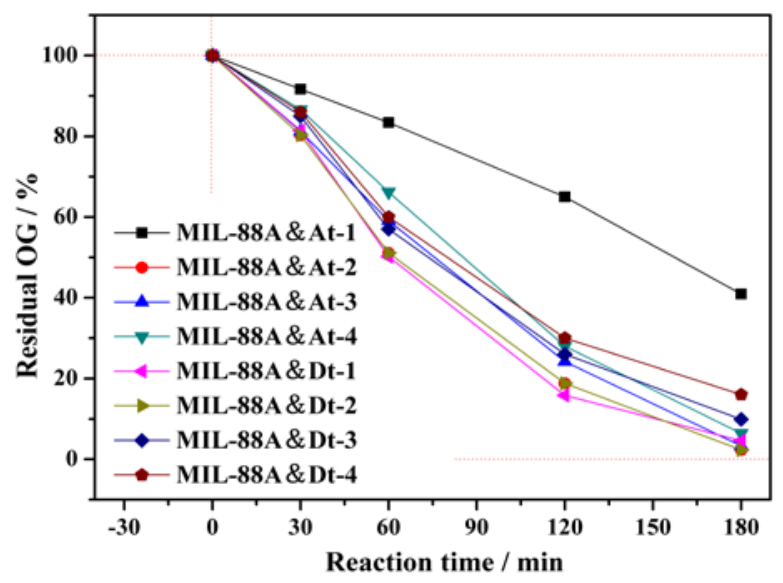

Fig. 3 The effect of activation temperature and drying time of MIL-88A on degradation of Orange G (Experimental conditions: $[\mathrm{OG}]=0.2 \mathrm{mmol} / \mathrm{L}, \mathrm{OG}: \mathrm{PS}=1: 60, \mathrm{MIL}-88 \mathrm{~A}$ dosage $=0.3 \mathrm{~g} / \mathrm{L}, \mathrm{T}=25^{\circ} \mathrm{C}$,

$$
\mathrm{pH}=3.26 \text {.) }
$$

\section{Degradation of DBP by MIL-88A}

As a highly toxic substance and due to its stable chemical structure and long chains, DBP is hard to destruct by conventional methods and more difficult to remove than OG. In this experiment, we take MIL-88A\&St-2/ St-3/ St-4 and MIL-88A\&Ct-2/ Ct-4/ Ct-6 as an example to study the catalytic performance on degradation of DBP. The results showed in Fig.4, MIL-88A\&St-3 and MIL-88A\&Ct-2 were the same samples. Comparing the degradation curve of OG by the same catalyst, there exist similar rules, and the removal rate of pollutants were found in the following order: MIL-88A\&St-3/Ct-2>St-2>St-4>Ct-4>Ct-6. After reaction for 8 hours, the optimal degradation rate of DBP can reach up to $76.4 \%$ catalyze by MIL-88A\&St-3/Ct-2, while the worst was only $41.2 \%$ activate by MIL-88A\&Ct-6. This result confirmed that the catalyst MIL-88A also can catalyze the degradation of DBP effectively as long as a suitable synthesis condition was selected. 


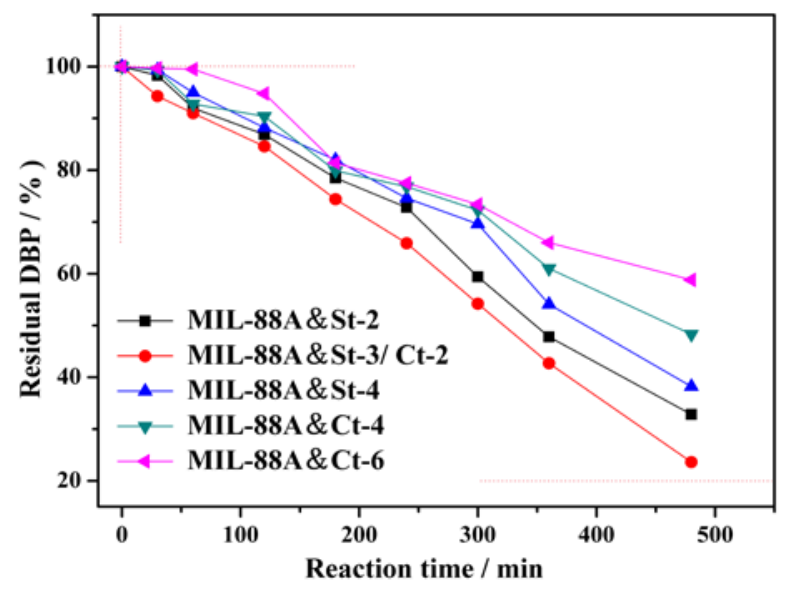

Fig. 4 Degradation of DBP by MIL-88A (Experimental conditions: [DBP] =5mg/L, DBP: PS=1:200, MIL-88A dosage $=0.3 \mathrm{~g} / \mathrm{L}, \mathrm{T}=25^{\circ} \mathrm{C}, \mathrm{pH}=3.26$.)

\section{Conclusion}

In brief, this article has mainly studied the effect of preparation conditions of MIL-88A on the degradation of OG, including raw materials proportion, synthesis/ activation temperature and crystallization/ drying time. The results showed that with the change of the proportion of Fecl $_{3} .6 \mathrm{H}_{2} \mathrm{O} /$ Fumaric acid, it was all not conducive to its performance whether it was higher or lower and the most suitable ratio was $n\left(\mathrm{Fecl}_{3} \cdot 6 \mathrm{H}_{2} \mathrm{O} /\right.$ Fumaric acid $)=1: 1$. Moreover, as the synthesis temperature increased from $45^{\circ} \mathrm{C}$ to $145^{\circ} \mathrm{C}$ and the crystallization time raised from $1 \mathrm{~h}$ to $12 \mathrm{~h}$, the catalytic activity of MIL-88A all showed a trend of rising and then decreasing mainly due to the difference of $\mathrm{S}_{\mathrm{BET}}$, dissolved quantity of $\mathrm{Fe}$ and pore volume. Another set of variables, activation temperature and drying time of MIL-88A, also have make a great difference to the degradation rate of OG, the activation temperature of $100^{\circ} \mathrm{C}$ could be the best choice to make the samples desiccation because of high relative content of $\mathrm{Fe}^{\mathrm{II}} / \mathrm{Fe}^{\mathrm{III}}$. While with the ascending of drying time of the catalyst, it would only cause an inhibition on the activation reaction. Furthermore, this study represents the first attempt to use the MIL-88A to activate PS for degrading DBP, which was a recognized noxious and undegraded organic pollutants. And the optimal removal rate of DBP can reach up to $76.4 \%$ catalyze by MIL-88A\&St-3/Ct-2, so the catalyst MIL-88A with the most suitable preparation conditions can be a high efficient catalyst on contaminants degradation.

\section{Acknowledgements}

This study was funded by National Natural Science Foundation of China (Grant No. 31570568, 31670585), State key laboratory of Pulp and Paper Engineering in China (No. 201535), Science and Technology Planning Project of Guangzhou City, China (No. 201607010079, 201607020007), Science and Technology Planning Project of Guangdong Province, China (No. 2016A020221005).

\section{References}

[1] N. Stock, S. Biswas, Synthesis of Metal-Organic Frameworks (MOFs): Routes to Various MOF Topologies, Morphologies, and Composites, Chem. Rev. 112 (2011) 933-969.

[2] C. Janiak, J. K. Vieth, MOFs, MILs and more: concepts, properties and applications for porous coordination networks (PCNs), New J. Chem. 34 (2010) 2366-2388. 
[3] U. Mueller, M. Schubert, F. Teich, H. Puetter, K. Schierle-Arndt, J. Pastre, Metal-organic frameworks-prospective industrial applications, J. Mater. Chem. 16 (2006) 626-636.

[4] J. R. Li, Y. Ma, M. C. McCarthy, J. Sculley, J. Yu, H. K. Jeong, P. B. Balbuena, H. C. Zhou, Carbon dioxide capture-related gas adsorption and separation in metal-organic frameworks, Coord. Chem. Rev. 255 (2011) 1791-1823.

[5] J. W. Yoon, S. H. Jhung, Y. K. Hwang, S. M. Humphrey, P. T. Wood, J. S. Chang, Gas-Sorption Selectivity of CUK-1: A Porous Coordination Solid Made of Cobalt ( II ) and Pyridine-2,4-Dicarboxylic Acid, Adv. Mater. 19 (2007) 1830-1834.

[6] J. R. Li, R. J. Kuppler, H. C. Zhou, Selective gas adsorption and separation in metal-organic frameworks, Chem. Soc. Rev. 38 (2009) 1477-1504.

[7] J. Lee, O. K. Farha, J. Roberts, K. A. Scheidt, S. T. Nguyen, J. T. Hupp, Metal-organic framework materials as catalysts, Chem. Soc. Rev. 38 (2009) 1450-1459.

[8] J. Gascon, A. Corma, F. Kapteijn, F. X. Llabr'es i Xamena, Metal Organic Framework Catalysis: Quo vadis, ACS Catal. (2013) 361-378.

[9] A. Corma, H. Garc' 1a, F. X. Llabr' es i Xamena, Engineering Metal Organic Frameworks for Heterogeneous Catalysis, Chem. Rev. 110 (2010) 4606-4655.

[10] L. G. Qiu, Z. Q. Li, Y. Wu, W. Wang, T. Xu, X. Jiang, Facile synthesis of nanocrystals of a microporous metal-organic framework by an ultrasonic method and selective sensing of organoamines, Chem. Commun. (2008) 3642-3644.

[11] P. Horcajada, T. Chalati, C. Serre, B. Gillet, C. Sebrie, T. Baati, J. F. Eubank, D. Heurtaux, P. Clayette, C. Kreuz, J. S. Chang, Y. K. Hwang, V. Marsaud, P. N. Bories, L. Cynober, S. Gil, G. Ferey, P. Couvreur, R. Gref, Porous, metal-organic-framework nanoscale carriers as a potential platform for drug delivery and imaging, Nat.Mater. 9 (2010) 172-178.

[12] Y. Cui, Y. Yue, G. Qian, B. Chen, Luminescent Functional Metal-Organic Frameworks, Chem. Rev. 112 (2011) 1126-1162.

[13] F. Ke, L. G. Qiu, Y. P. Yuan, F. M. Peng, X. Jiang, A. J .Xie, Y. H. Shen, J. F. Zhu, Thiol-functionalization of metal-organic framework by a facile coordination-based postsynthetic strategy and enhanced removal of $\mathrm{Hg}^{2+}$ from water, J. Hazard. Mater. 196 (2011) 36-43.

[14] X. Zhao, D. Liu, H. Huang, W. Zhang, Q. Yang, C. Zhong, The stability and defluoridation performance of MOFs in fluoride solutions, Microporous Mesoporous Mater. 185 (2014) 72-78.

[15] L. Li, J. C. Li, Z. Rao, G. W. Song, B. Hu, Metal Organic Framework $\left[\mathrm{Cu}_{3}(\mathrm{BTC})_{2}\left(\mathrm{H}_{2} \mathrm{O}\right)_{3}\right]$ for the adsorption of methylene blue from aqueous solution, Desalin. Water Treat. (2013) 1-7.

[16] J. Q. Jiang, C. X. Yang, X. P. Yan, Zeolitic Imidazolate Framework-8 for Fast Adsorption and Removal of Benzotriazoles from Aqueous Solution, ACS Appl. Mater. Interfaces. 5 (2013) 9837-9842.

[17] H. Lv, H. Y. Zhao, T. C. Cao, L. Qian, Y. B. Wang, G. H. Zhao, Efficient degradation of high concentration azo-dye wastewater by heterogeneous Fenton process with iron-based metal-organic framework, Journal of Molecular Catalysis A: Chemical. 400 (2015) 81-89.

[18] G. H. Cui, C. H. He, C. H. Jiao, J. C. Geng, V. A. Blatov, Two metal-organic frameworks with unique high-connected binodal network topologies: synthesis, structures, and catalytic properties, 
Cryst. Eng. Comm. 14 (2012) 4210-4216.

[19] K. Y. Andrew Lin, H. A. Chang, Zeolitic Imidazole Framework-67 (ZIF-67) as a heterogeneous catalyst to activate peroxymonosulfate for degradation of Rhodamine B in water, J. Taiwan. Inst. Chem. E. 53 (2015) 40-45.

[20] H. X. Li, J. Q. Wan, Y. W. Ma, Y. Wang, X. Chen, Z. Y. Guan, Degradation of refractory dibutyl phthalate by peroxymonosulfate activated with novel catalysts cobalt metal-organic frameworks: Mechanism, Performance, and Stability, J. Hazard. Mater. 318 (2016) 154-163.

[21] J. J. Du, Y. P. Yuan, J. X. Sun, F. M. Peng, X. Jiang, L. G. Qiu, A. J. Xie, Y. H. Shen, J. F. Zhu, New photocatalysts based on MIL-53 metal-organic frameworks for the decolorization of methylene blue dye, J. Hazard. Mater. 190 (2011), 945-951.

[22] L. H. Ai, C. Zhang, L. Li, J. Jiang, Iron terephthalate metal-organic framework: revealing the effective activation of hydrogen peroxide for the degradation of organic dye under visible light irradiation, Appl. Catal. B. (2014) 148-149, 191-200.

[23] S. Bhattacharjee, J. S. Choi, S. T. Yang, S. B. Choi, J. Kim, W. S. Ahn, Solvothermal Synthesis of Fe-MOF-74 and Its Catalytic Properties in phenol Hydroxylation, J. Nanosci. Nanotechnol. 10 (2010) 135-141.

[24] T. A. Vu, G. H. Le, C. D. Dao, L. Q. Dang, K. T. Nguyen, P. T. Dang, H. T. K. Tran, Q. T. Duong, T. V. Nguyen, G. D. Lee, Isomorphous substitution of Cr by Fe in MIL-101 framework and its application as a novel heterogeneous Photo-Fenton catalyst for reactive dye degradation, RSC Adv. 4 (2014) 41185-41194.

[25] G. P. Yang, X. K. Zhao, X. J. Sun, X. L. Lu, Oxidative degradation of diethyl phthalate by photochemically-enhanced Fenton reaction, J. Hazard. Mater B. 126 (2005) 112-118.

[26] J. L. Lyche, A. C. Gutleb, A. Bergman, G. S.Eriksen, A.J. Murk, E. Ropstad, M. Saunders, J. U. Skaare, Reproductive and Developmental Toxicity of Phthalates, J. Toxicol. Environ. Health, Part B: Critical Reviews. 12 (2009) 225-249.

[27] J. Ejlertsson, M. Alnervik, S. Jonsson, B. H. Svensson, Influence of water solubility, side-chain degradability, and side-chain structure on the degradation of phthalic acid esters under methanogenic conditions, Environ. Sci. Technol. 31 (1997) 2761-2764.

[28] J. M. Wang, J. Q. Wan, Y. W. Ma, Y. Wang, M. J. Pu, Z. Y. Guan, Metal-organic frameworks MIL-88A with suitable synthesis conditions and optimal dosage for effective catalytic degradation of Orange G through persulfate activation, RSC Adv. 6 (2016) 112502-112511.

[29] M. J. Pu, Y. W. Ma, Y. Wang, J. M. Wang, M. L. Brusseau, Activation performance and mechanism of a novel heterogeneous persulfate catalyst: Metal Organic Framework MIL-53(Fe) with $\mathrm{Fe}^{\mathrm{II}} / \mathrm{Fe}^{\mathrm{III}}$ mixed-valence coordinative unsaturated iron center, Catal. Sci. Technol. 7 (2017) 1129-1140.

[30] M. Elo1'sa Medina, Y. Dumont, J. Grene'che, F. Millange, $\mathrm{Fe}^{\mathrm{III}} / \mathrm{Fe}^{\mathrm{II}}$ regular charge order in metal-organic framework, Chemi. Commun. 46 (2010) 7987-7989. 